\title{
A fuzzy semi-infinite optimization problem
}

\section{Abd El-Monem A Megahed*}

${ }^{*}$ Correspondence:

a_megahed15@yahoo.com

Permanent address: Department of

Basic Science, Faculty of Computers

and Informatics, Suez Canal

University, Ismalia, Egypt

Present address: Department of

Mathematics, College Of Science,

Majmaah University, Al-Zulfi, KSA

\begin{abstract}
In this paper, we present a fuzzy semi-infinite optimization problem. Moreover, we will deduce the Fritz-John and Kuhn-Tucker necessary conditions of this problem. Finally, a numerical example is given to illustrate the results.
\end{abstract}

MSC: 90C34; 90C05; 90C70; 90C30; 90C46

Keywords: fuzzy; semi-infinite; optimization; necessary conditions

\section{Introduction}

In many practical problems, we might have information containing some uncertainty, which is treated in this paper as fuzzy information; the considered semi-infinite optimization problem with fuzzy information is a fuzzy semi-infinite optimization problem.

Fuzzy set theory was introduced into conventional linear programming by Zimmermann [1], fuzzy mathematical programming was presented in [2], and fuzzy programming and linear programming with several objective functions were presented by [3].

Optimality conditions of a nonlinear programming problem with fuzzy parameters are established, and a fuzzy function is defined, with its differentiability, convexity, and some important properties being studied in [4]; the fuzzy solution of optimization problems and the incentive solution of the optimization problems are presented which explain that the solution of optimization problems is a generalization of the solutions in the case of crisp optimization problems [5]. As regards fuzzy mathematical programming: theory, application, and an extension are presented in [6].

This paper is organized as follows: In Section 2, the formulation of the problem of semiinfinite optimization is considered. In Section 3, the main section of the paper, we will study a fuzzy semi-infinite optimization problem, and the Fritz-John and Kuhn-Tucker necessary conditions. Finally, the conclusion is drawn in Section 5.

\section{A semi-infinite programming problem}

A semi-infinite programming problem is an optimization problem in which finitely many variables appear with infinitely many constraints $[7,8]$, and we consider a generalized semi-infinite optimization problem (GSIP) $[9,10]$ of the form

$$
\left.\begin{array}{r}
\min _{x} f(x) \text { s.t. } x \in M, \\
\text { M }=\left\{\begin{array}{c}
x \in R^{n} / h_{i}(x)=0, i \in I, G(x, y) \geq 0 \\
\text { for all } y \in Y(x)
\end{array}\right\}, \\
\left.y \in R^{r} / u_{k}(x, y)=0, k \in K, v_{l}(x, y) \geq 0, l \in L\right\} .
\end{array}\right\}
$$


$I, K$, and $L$ are finite index sets with $|l|<n$ and $|K|<r$ (where $|\cdot|$ denotes the cardinality), all appearing functions are real valued and continuously differentiable, and the set $Y(\bar{x})$ is compact for each $\bar{x} \in R^{n}$, and the set-valued mapping $Y: x \in R^{n} \rightarrow Y(x) \subset R^{n}$ is upper semi-continuous at each $\bar{x} \in R^{n}$.

For the special case that the set $Y(x)=Y$ does not depend on the variable $x$, this problem is a common semi-infinite problem (SIP). The generalized semi-infinite and bi-level optimization problem are presented by Stein and Still [11]. Bi-level problems are of the following form.

(BL):

$$
\begin{gathered}
\min _{x} f(x) \text { s.t. } x \in M \text { and } y \text { is a solution of } \\
\min _{y} G(x, y) \\
\text { s.t. } y \in Y(x) .
\end{gathered}
$$

The generalized semi-infinite programming on generic local minimizers was introduced by Gunzel et al. [12]. The feasible set in generalized semi-infinite optimization is presented by Jongen et al. in [13]. Furthermore, the linear and linearized generalized semi-infinite optimization problems were introduced by Rukmann [14]. In [15], a first-order optimality condition in generalized semi-infinite programming is introduced. Still discussed the optimality conditions for generalized semi-infinite programming problems in [16].

\section{A fuzzy semi-infinite programming problem}

\subsection{Problem formulation}

A fuzzy semi-infinite programming problem is defined as

$$
\left.\begin{array}{r}
\widetilde{\min _{x}} f(x) \text { s.t. } x \in M, \\
\text { where } M=\left\{\begin{array}{r}
x \in R^{n} / h_{i}(x)=0, i \in I, G(x, y) \geq 0 \\
\text { for all } y \in Y(x)
\end{array}\right\}, \\
(x)=\left\{y \in R^{r} / u_{k}(x, y)=0, k \in K, v_{l}(x, y) \geq 0, l \in L\right\} .
\end{array}\right\}
$$

All functions of this problem have the same properties as the problem (2.1). For $\bar{x} \in M$ denote the index sets of the active inequality constraints by

$$
\begin{aligned}
& Y_{0}(\bar{x})=\{y \in Y(\bar{x}) / G(\bar{x}, y)=0\}, \\
& L_{0}(\bar{x}, \bar{y})=\left\{l \in L / v_{l}(\bar{x}, \bar{y})=0 \text { for } \bar{y} \in Y(\bar{x})\right\} .
\end{aligned}
$$

\subsection{Lower level problem}

Consider the following lower level problem:

$$
\left.\begin{array}{c}
\underset{y}{\min _{y}} G(\bar{x}, y) \\
\text { s.t. } y \in Y(\bar{x}) .
\end{array}\right\}
$$

The fuzzy requirements of the lower level problem (3.3) can be quantified by electing a membership function $\mu(G(\bar{x}, y))$ (Figure 1) which is differentiable in the open interval 
Figure 1 The membership function $\mu(G(x, y))$.

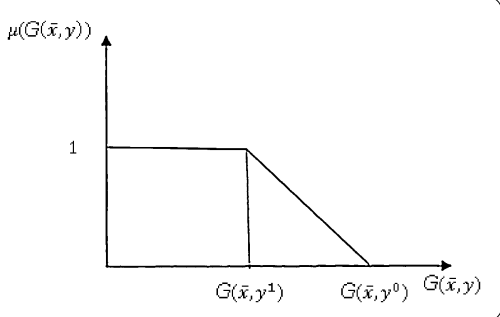

$G\left(\bar{x}, y^{1}\right)<G(\bar{x}, y)<G\left(\bar{x}, y^{0}\right)$, where $\mu(G(\bar{x}, y))$ is defined by

$$
\mu(G(\bar{x}, y))= \begin{cases}1, & G(\bar{x}, y) \leq G\left(\bar{x}, y^{1}\right), \\ \frac{G(\bar{x}, y)-G\left(\bar{x}, y^{0}\right)}{G\left(\bar{x}, y^{1}\right)-G\left(\bar{x}, y^{0}\right)}, & G\left(\bar{x}, y^{1}\right) \leq G(\bar{x}, y) \leq G\left(\bar{x}, y^{0}\right), \\ 0, & G(\bar{x}, y) \geq G\left(\bar{x}, y^{0}\right),\end{cases}
$$

where $G\left(\bar{x}, y^{0}\right)$ and $G\left(\bar{x}, y^{1}\right)$ denote the values of the objective function of the lower level problem (3.3) with the degree of the membership function 0 and 1, respectively, i.e., $G\left(\bar{x}, y^{0}\right)$ is an undesirable value and $G\left(\bar{x}, y^{1}\right)$ is a desirable value of the objective function $G(\bar{x}, y)$.

Definition 1 The $\alpha$-level set of the fuzzy goal $G(\bar{x}, y)$ is defined as the ordinary set $L_{\alpha}(G(\bar{x}, y))$; for the value of $G(\bar{x}, y)$ the degree of its membership function exceeds the level $\alpha$, i.e.

$$
L_{\alpha}(G(\bar{x}, y))=\{G(\bar{x}, y) / \mu(G(\bar{x}, y)) \geq \alpha, \alpha \in(0,1)\},
$$

where $\alpha$ is the least acceptable degree of the required value. For certain degrees $\alpha$ the problem (3.3) can be transformed into the following equivalent form:

$$
\left.\begin{array}{c}
\min _{y} G(\bar{x}, y) \\
\text { s.t. } y \in Y(\bar{x}) \\
\mu(G(\bar{x}, y)) \geq \alpha .
\end{array}\right\}
$$

If $\bar{x} \in M$ and $\bar{y} \in Y_{0}(\bar{x})$, then $\bar{y}$ is a minimizer of the problem (3.5).

By the Fritz-John conditions there exist coefficients $\bar{\lambda}, \bar{\beta}=\left(\bar{\beta}_{k}, k \in K\right), \bar{\gamma}=\left(\bar{\gamma}_{l}, l \in\right.$ $\left.L_{0}(\bar{x}, \bar{y})\right)$, and $\bar{v}$ satisfying

$$
\begin{aligned}
& D_{y} \mathcal{L}^{(\bar{x}, \bar{y})}(\bar{x}, \bar{y}, \bar{\lambda}, \bar{\beta}, \bar{\gamma}, \bar{v})=0 \text { and } \bar{\lambda}+\bar{v}+\sum_{k \in K}\left|\bar{\beta}_{k}\right|+\sum_{l \in L_{0}(\bar{x}, \bar{y})} \bar{\gamma}_{l}=1, \\
& \bar{\lambda} \geq 0, \bar{\gamma}_{l} \geq 0, \bar{v} \geq 0, l \in L_{0}(\bar{x}, \bar{y})
\end{aligned}
$$

where

$$
\mathcal{L}^{(\bar{x}, \bar{y})}(x, y, \lambda, \beta, \gamma, v)=\lambda G(\bar{x}, y)-v(\mu(G(\bar{x}, y))-\alpha)-\sum_{k \in K} \beta_{i} u_{k}(x, y)-\sum_{l \in L_{0}(\overline{\bar{x}}, \bar{y})} \gamma_{l} \nu_{l} .
$$

In other words, for $\bar{x} \in M$ and $\bar{y} \in Y_{0}(\bar{x})$ the $\operatorname{set} F(\bar{x}, \bar{y})=\left\{(\lambda, \beta, \gamma, v) \in R \times R^{|k|} \times R^{\left|L_{0}(\bar{x}, \bar{y})\right|} \times\right.$ $R /(\lambda, \beta, \gamma, v)$ satisfies (3.6)\} is nonempty and, furthermore, is also compact. 


\section{Fritz-John conditions}

Firstly, we will give some lemmas, definitions, and a proposition which will be used in the proof of the Fritz-John conditions.

Lemma 2 [9] Let $x^{t} \in M, y^{t} \in Y\left(x^{t}\right)$ and $x^{t} \rightarrow \bar{x}$. Then the set $\left\{\left\|y^{t}\right\|, t \in N\right\}$ is bounded, and $\bar{y} \in Y(\bar{x})$ whenever $y^{t} \rightarrow \bar{y}$.

Lemma 3 [9] Let $l=\phi$, and for $\bar{x} \in M$ let $Y_{0}(\bar{x})=\phi$. Then $\bar{x}$ is an interior point of $M$.

Definition 4 For $\bar{x} \in M$ define

$$
V(\bar{x})=\bigcup_{\bar{y} \in Y_{0}(\bar{x})}\left\{D_{x} \mathcal{L}^{(\bar{x}, \bar{y})}(\bar{x}, \bar{y}, \lambda, \beta, \gamma, \nu) /(\alpha, \beta, \gamma) \in F(\bar{x}, \bar{y})\right\} .
$$

Lemma 5 [9] Let $\bar{x} \in M$. Then the set $V(\bar{x})$ is compact.

Definition 6 [17] For a set $V$, we $\operatorname{define} \operatorname{Conv}(V)$ to denote the convex hull of $V$, i.e. $x \in \operatorname{Conv}(V)$ if and only if

$$
x=\sum_{i=1}^{n} \lambda_{i} x_{i}, \quad x_{i} \in V, \sum_{i=1}^{n} \lambda_{i}=1, \lambda_{i} \geq 0,
$$

i.e. $\operatorname{Conv}(V)$ consists of all finite convex combinations of the elements of $V$.

Lemma 7 [18] Let $V \subset R^{n}$ be a nonempty compact set. Then there exists a $\xi \in R^{n}$ with $s^{T} \xi>0$ for all $s \in V$ if and only if $0 \notin \operatorname{Conv}(V)$.

Lemma 8 [19] Let $I_{1}, I_{2}$ be finite index sets and $s^{i} \in R^{n}, i \in I_{1}$, and $z^{j} \in R^{n}, j \in I_{2}$. Then either (i) or (ii) holds.

(i) There are real numbers $a_{i}, i \in I_{1}, b_{j} \geq 0, j \in I_{2}$, satisfying

$$
\begin{aligned}
& \sum_{i \in I} a_{i} s^{i}+\sum_{j \in I_{2}} b_{j} z^{j}=0, \\
& \sum_{i \in I_{1}}\left|a_{i}\right|+\sum_{j \in I_{2}} b_{j}=1 .
\end{aligned}
$$

(ii) The set $\left\{s^{i}, i \in I_{1}\right\}$ is linearly independent and there exists $a \xi \in R^{n}$ with

$$
\left(s^{i}\right)^{T} \xi=0, \quad i \in I_{1}, \quad\left(z^{j}\right)^{T} \xi>0, \quad j \in I_{2} .
$$

Proposition 9 [9] For $t \in R$ and $y \in R^{r}$, we define the following functions:

$$
\begin{aligned}
& \bar{u}_{k}(t, y)=u_{k}(\bar{x}+t \xi, y), \quad k \in K, \\
& \bar{v}_{l}(t, y)=v_{l}(\bar{x}+t \xi, y), \quad l \in L, \\
& \bar{G}(t, y)=G(\bar{x}+t \xi, y) .
\end{aligned}
$$

Then the following conditions are satisfied. 
Figure 2 The membership function $\mu(f(x))$.

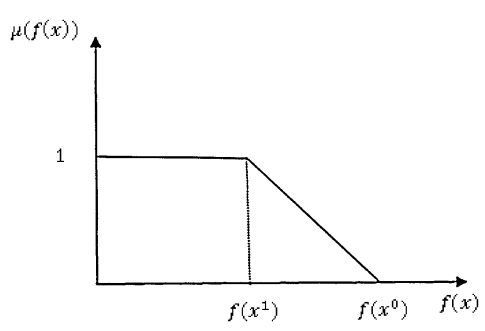

(i) The set $\left\{D \bar{u}_{k}(0, \bar{y}), k \in K\right\}\left(=\left\{\left[D_{x} u_{k}(\bar{x}, \bar{y}) \xi, D_{y} u_{k}(\bar{x}, \bar{y})\right], k \in K\right\}\right)$ is linearly independent.

(ii) There is a $w \in R^{r+1}$ satisfying

$$
\left.\begin{array}{r}
D \bar{G}(0, \bar{y}) w>0, \\
D \bar{u}_{k}(0, \bar{y}) w=0, k \in K, \\
D \bar{v}_{k}(0, \bar{y}) w<0, l \in L_{0}(E \bar{x}, \bar{y}) .
\end{array}\right\}
$$

The fuzzy requirements of the problem (3.1) can be quantified by electing a membership function $\mu(f(x))$ (Figure 2) which is differentiable in the open interval $f\left(x^{1}\right)<f(x)<f\left(x^{0}\right)$ where $\mu(f(x))$ is defined by

$$
\mu(f(x))= \begin{cases}1, & f(x) \leq f\left(x^{1}\right) \\ \frac{f(x)-f\left(x^{1}\right)}{f\left(\bar{x}^{0}\right)-f\left(\bar{x}^{1}\right)}, & f\left(x^{1}\right) \leq f(x) \leq f\left(x^{0}\right) \\ 0, & f(x) \geq f\left(x^{0}\right),\end{cases}
$$

where $f\left(x^{0}\right)$ and $f\left(x^{1}\right)$ denote the values of the objective function of the problem (3.1) with the degree of membership function 0 and 1 , respectively, i.e., $f\left(x^{0}\right)$ is an undesirable value and $f\left(x^{1}\right)$ is a desirable value of the objective function $f(x)$. For a certain degree $\alpha_{1}$ the defuzzification of the problem (3.1) is

$$
\begin{aligned}
& \min _{x} f(x) \text { s.t. } x \in M, \\
& \text { where } M=\left\{\begin{array}{c}
x \in R^{n} / h_{i}(x)=0, i=1, \ldots, m, G(x, y) \geq 0 \\
\text { for all } y \in Y(x)
\end{array}\right\}, \\
& Y(x)=\left\{y \in R^{r} / u_{k}(x, y)=0, k \in K, v_{l}(x, y) \geq 0, l \in L\right\}, \\
& \mu(f(x)) \geq \alpha_{1}, \quad \alpha_{1} \in(0,1), \\
& \mu(G(\bar{x}, y)) \geq \alpha, \quad \alpha \in(0,1) .
\end{aligned}
$$

Theorem 10 Let $\bar{x}$ be a local minimizer of the problem (4.7). Then either $Y_{0}(\bar{x}) \neq \phi$ and there exist

$$
\left.\begin{array}{r}
y^{j} \in Y_{0}(\bar{x}), j=1, \ldots, p, \\
\left(\lambda^{j}, \beta^{j}, \gamma^{j}, v^{j}\right) \in F\left(\bar{x}, y^{j}\right), j=1, \ldots, p, \\
k \geq 0, \eta \geq 0, \rho_{i}, i \in I, \zeta_{j} \geq 0, j=1, \ldots, p,
\end{array}\right\}
$$


satisfying

$$
\left.\begin{array}{r}
k+\eta+\sum_{i \in I}\left|\rho_{i}\right|+\sum_{j=1}^{p} \zeta_{j}>0 \\
\text { as well as } \\
k D f(\bar{x})-\eta D\left(\mu\left(f(\bar{x})-\alpha_{1}\right)\right)-\sum_{i \in I} \rho_{i} D h_{i}(x) \\
-\sum_{j=1}^{p} \zeta_{j} D_{x} \mathcal{L}^{\left(\bar{x}, y^{j}\right.}\left(\bar{x}, y^{j}, \lambda^{j}, \beta^{j}, \gamma^{j}, v^{j}\right)=0,
\end{array}\right\}
$$

or $Y_{0}(\bar{x})=\phi$ and there exist $k \geq 0, \eta \geq 0, \rho_{i}, i \in I$, satisfying

$$
\left.\begin{array}{r}
k+\eta+\sum_{i \in I}\left|\rho_{i}\right|>0 \\
k D f(\bar{x})-\eta D\left(\mu\left(f(\bar{x})-\alpha_{1}\right)\right)-\sum_{i \in I} \rho_{i} D h_{i}(x)=0
\end{array}\right\}
$$

Proof Let $\bar{x}$ be a local minimizer of the problem (4.7). We distinguish three cases.

Case 1: The set $\left\{D h_{i}(\bar{x}), i \in I\right\}$ is linearly dependent. Then we are done by choosing $k=0$, $\eta=0, \zeta=0$ in (4.9) (if $Y_{0}(\bar{x}) \neq \phi$ ), or $k=0, \eta=0$ in (4.10) (if $Y_{0}(\bar{x})=\phi$ ) as well as a linear combination $\sum_{i \in I} \rho_{i} D h_{i}(x)=0$ with $\sum_{i \in I}\left|\rho_{i}\right|>0$.

Case 2: There exists a $\bar{y} \in Y_{0}(\bar{x})$ and the set $\left\{D u_{k}(\bar{x}, \bar{y})=0, k \in K\right\}$ is linearly dependent. Then there exists a linear combination $\sum_{k \in K} \bar{\beta}_{k} D u_{k}(\bar{x}, \bar{y})=0, \sum_{k \in K}\left|\bar{\beta}_{k}\right|=1$ and therefore we have $\sum_{k \in K} \bar{\beta}_{k} D_{y} u_{k}(\bar{x}, \bar{y})=0,(0, \beta, 0,0) \in F(\bar{x}, \bar{y})$ (with $\bar{\beta}=\left(\bar{\beta}_{k}, k \in K\right)$ ), and

$$
D_{x} \mathcal{L}^{(\overline{\bar{x}}, \bar{y})}(\bar{x}, \bar{y}, 0,0, \beta, 0)=\sum_{k \in K} \bar{\beta}_{k} D u_{k}(\bar{x}, \bar{y})=0 .
$$

Case 3: Neither Case 1 nor Case 2 holds. Then the proof is similar to Theorem 1.1 in [9].

\section{A constraint qualification}

Definition 11 The Mangasarian-Fromovitz constraint qualification (MFCQ) of the problem (4.7) is said to hold at $\bar{x}$ if:

(1) the set $\left\{D h_{i}(\bar{x}), i \in I\right\}$ is linearly independent and

(2) there exists a $\varpi \in R^{n}$ such that

$$
\left.\begin{array}{r}
\text { for all } i \in I: D h_{i}(\bar{x}) \varpi=0, \\
\text { for all } \bar{y} \in Y_{0}(\bar{x}): D_{x} \mathcal{L}^{(\bar{x}, \bar{y})}(\bar{x}, \bar{y}, \bar{\lambda}, \bar{\beta}, \bar{\gamma}, \bar{v}) \varpi>0, \\
\text { for all }(\bar{\lambda}, \bar{\beta}, \bar{\gamma}, \bar{v}) \in F(\bar{x}, \bar{y}) .
\end{array}\right\}
$$

Theorem 12 Let $\bar{x}$ be a local minimizer of the problem (4.7) and (MFCQ) be satisfied. Then either $Y_{0}(\bar{x}) \neq \phi$ and there exist

$$
\left.\begin{array}{r}
y^{j} \in Y_{0}(\bar{x}), j=1, \ldots, p, \\
\left(\lambda^{j}, \beta^{j}, \gamma^{j}, v^{j}\right) \in F\left(\bar{x}, y^{j}\right), j=1, \ldots, p, \\
k \geq 0, \eta \geq 0, \rho_{i}, i \in I, \zeta_{j} \geq 0, j=1, \ldots, p,
\end{array}\right\}
$$

satisfying

$$
\left.\begin{array}{r}
k+\eta+\sum_{i \in I}\left|\rho_{i}\right|+\sum_{j=1}^{p} \zeta_{j}>0 \\
\text { as well as } \\
k D f(\bar{x})-\eta D\left(\mu\left(f(\bar{x})-\alpha_{1}\right)\right)-\sum_{i \in I} \rho_{i} D h_{i}(x) \\
\left.-\sum_{j=1}^{p} \zeta_{j} D_{x} \mathcal{L}^{\left(\bar{x}, y^{j}\right.}\right)\left(\bar{x}, y^{j}, \lambda^{j}, \beta^{j}, \gamma^{j}, \nu^{j}\right)=0,
\end{array}\right\}
$$


or $Y_{0}(\bar{x})=\phi$ and there exist $k \geq 0, \eta \geq 0, \rho_{i}, i \in I$, satisfying the set

$$
\left.\begin{array}{r}
k+\eta+\sum_{i \in I}\left|\rho_{i}\right|>0, \\
k D f(\bar{x})-\eta D\left(\mu\left(f(\bar{x})-\alpha_{1}\right)\right)-\sum_{i \in I} \rho_{i} D h_{i}(x)=0 .
\end{array}\right\}
$$

The proof is similar to the proof of Theorem 10 if we choose $k=1$.

\section{Example}

$\widetilde{\min } f(x)=\left(x_{1}-\frac{1}{4}\right)^{2}+x_{2}^{2}$

subject to $G(x, y)=y+x_{2}$,

$V(x, y)=x_{1}-y^{2}$.

The lower level problem is

$\widetilde{\min } G(x, y)=y+x_{2}$,

subject to $V(x, y)=x_{1}-y^{2}$.

The optimal solution of the crisp problem is $\left(x_{1}, x_{2}\right)=\left(\frac{1}{4}, 0\right), y=0$, and $(\lambda, \gamma)=(0,1)$; and $f^{1}\left(\frac{1}{4}, 0\right)=0, G^{1}(x, y)=0$.

Let $f^{0}(x)=1$ and $G^{0}(x, y)=1$ be undesirable values of the problem, the membership functions of $f$ and $G$ are defined by

$$
\begin{aligned}
& \mu(f)=\frac{f-f^{0}}{f^{1}-f^{0}}=-\left[\left(x_{1}-\frac{1}{4}\right)^{2}+x_{2}^{2}-1\right], \\
& \mu(G)=\frac{G-G^{0}}{G^{1}-G^{0}}=1-y-x_{2} .
\end{aligned}
$$

The new problem is

$$
\min f(x)=\left(x_{1}-\frac{1}{4}\right)^{2}+x_{2}^{2}
$$

subject to $G(x, y)=y+x_{2}$,

$$
\begin{aligned}
& V(x, y)=x_{1}-y^{2}, \\
& -\left[\left(x_{1}-\frac{1}{4}\right)^{2}+x_{2}^{2}-1\right] \geq \alpha_{1}, \quad \alpha_{1} \in(0,1), \\
& 1-y-x_{2} \geq \alpha_{2}, \quad \alpha_{2} \in(0,1) .
\end{aligned}
$$

The lower level problem is

$$
\begin{aligned}
& \min G(x, y)=y+x_{2}, \\
& V(x, y)=x_{1}-y^{2}, \\
& 1-y-x_{2} \geq \alpha_{2},
\end{aligned}
$$


then

$$
\begin{aligned}
& \mathcal{L}\left(x, y, \alpha_{1}, \gamma_{3}, \gamma_{5}\right)=\lambda_{1}\left(y+x_{2}\right)-\gamma_{1}\left(x_{1}-y^{2}\right)-\gamma_{2}\left(-y-x_{2}+1-\alpha_{2}\right), \\
& D_{y} \mathcal{L}\left(x, y, \alpha_{1}, \gamma_{3}, \gamma_{5}\right)=\lambda_{1}+2 y \gamma_{1}+\gamma_{2}=0, \quad \text { then } y=-\frac{\lambda_{1}+\gamma_{2}}{2 \gamma_{1}}, \lambda_{1}+\gamma_{1}+\gamma_{2}=1 .
\end{aligned}
$$

Since

$$
k D f(\bar{x})-\gamma_{3} D\left(\mu\left(f(\bar{x})-\alpha_{1}\right)\right)-\beta_{1} D_{x} \mathcal{L}\left(x, y, \lambda_{1}, \gamma_{1}, \gamma_{2}\right)=0,
$$

we have

$$
\begin{aligned}
& 2 k\left(x_{1}-\frac{1}{4}\right)+2 \gamma_{3}\left(x_{1}-\frac{1}{4}\right)+\beta_{1} \gamma_{1}=0, \quad x_{1}=\frac{1}{4}-\frac{\beta_{1} \gamma_{1}}{2 k+2 \gamma_{3}}, \\
& 2 k x_{2}+2 \gamma_{3} x_{2}-\beta_{1}\left(\lambda_{1}+\gamma_{2}\right)=0, \quad x_{2}=\frac{\beta_{1}\left(\lambda_{1}+\gamma_{2}\right)}{2 k+2 \gamma_{3}} ;
\end{aligned}
$$

the solution is

$$
\left\{\left[\begin{array}{c}
\beta_{1}=1.0, k=0.0, \gamma_{3}=0.583, \lambda_{1}=0.2, \\
\gamma_{1}=0.7, \gamma_{2}=0.1, \\
\alpha_{1}=0.21706, \alpha_{2}=0.86272, x_{2}=0.257, \\
x_{1}=0.0457, y=-0.21428
\end{array}\right], f=0.107662, G=0.043\right\} \text {. }
$$

\section{Conclusion}

In this work, we discussed a fuzzy semi-infinite optimization problem, by considering that the minimum of the objective function is fuzzy ( $\widetilde{\min })$. The Fritz-John conditions and the constraint qualification are discussed for this problem. Finally, an illustrative example is given to clarify the results.

\section{Competing interests}

The author declares that they have no competing interests.

\section{Acknowledgements}

The author wants to express his deep thanks and his respect to his faculty, colleagues, the Journal, and Prof. Dr. Rachel M Bernales of the Journal of Editorial office. Also the author wants to express his thanks and respect to Jane Doe who provided medical writing services on behalf of XYZ pharmaceuticals Ltd.

Received: 11 July 2014 Accepted: 5 November 2014 Published: 19 Nov 2014

\section{References}

1. Zimmermann, H-J: Description and optimization of fuzzy systems. Int. J. Gen. Syst. 2, 209-215 (1976)

2. Zimmermann, H-J: Fuzzy mathematical programming. Comput. Oper. Res. 10, 291-298 (1983)

3. Zimmermann, H-J: Fuzzy programming and linear programming with several objective functions. Fuzzy Sets Syst. 1, 45-55 (1978)

4. Cantão, LAP, Yamakami, A: Nonlinear programming with fuzzy parameters: theory and applications. In: Mohammedian, M (ed.) Proceedings of CIMCA 2003. ISBN:1740880684

5. Jameed, AF, Sadeghi, A: Solving nonlinear programming problem in fuzzy environment. Int. J. Contemp. Math. Sci. 7(4), 159-170 (2012)

6. Luhandjula, MK: Fuzzy mathematical programming: theory, applications and extension. J. Uncertain Syst. 1(2), 124-136 (2007)

7. Reemsten, R, Ruckmann, J-J (eds.): Semi-Infinite Programming. Kluwer Academic, Boston (1998)

8. Hettich, R, Kortank, KO: Semi-infinite programming: theory methods, and applications. SIAM Rev. 35, 380-429 (1993)

9. Jongen, HT, Ruckmann, J-J, Stein, O: Generalized semi-infinite optimization: a first order optimality condition and examples. Math. Program. 83, 145-158 (1998)

10. Weber, G-W: Generalized semi-infinite optimization: on some foundation. Vyčisl. Tehnol. 4, 41-61 (1999) 
11. Stein, O, Still, G: On generalized semi-infinite optimization and bi-level optimization. Eur. J. Oper. Res. 142, 444-462 (2002)

12. Gunzel, H, Jongen, HT, Stein, O: Generalized semi-infinite programming: on generic local minimizers. J. Glob. Optim. 42(3), 413-421 (2008)

13. Jongen, HT, Twilt, F, Weber, G-W: Semi infinite optimization: structure and feasibility of the feasible set. J. Optim. Theory Appl. 72, 529-552 (1992)

14. Rukmann, JJ: On linear and liberalized generalized semi-infinite optimization problem. Ann. Oper. Res. 101, 191-208 (2001)

15. Ruckmann, J-J, Shapiro, A: First-order optimality conditions in generalized semi-infinite programming. J. Optim. Theory Appl. 101, 677-691 (1999)

16. Stein, O, Still, G: On optimality conditions for generalized semi- infinite programming problems. J. Optim. Theory Appl. 104, 443-458 (2000)

17. Bazaraa, MS, Shetty, CM: Nonlinear Programming Theory and Algorithms. Wiley, New York (1979)

18. Cheney, EW: Introduction to Approximation Theory. McGraw-Hill, New York (1966)

19. Jongen, HT, Jonker, P, Twilt, F: Nonlinear Optimization in $R^{n}$. II. Transversality, Flows, Parametric Aspects. Peter Lang, Frankfurt am Main (1986)

Cite this article as: Megahed: A fuzzy semi-infinite optimization problem. Journal of Inequalities and Applications 2014, 2014:457

\section{Submit your manuscript to a SpringerOpen ${ }^{\ominus}$ journal and benefit from:}

- Convenient online submission

Rigorous peer review

- Immediate publication on acceptance

- Open access: articles freely available online

- High visibility within the field

- Retaining the copyright to your article 